\title{
Division of labour and terminal differentiation in a novel Bacillus thuringiensis strain
}

\author{
Chao Deng ${ }^{1,2,3}$, Leyla Slamti ${ }^{2,3}$, Ben Raymond ${ }^{4}$, Guiming Liu ${ }^{5}$, Christelle Lemy ${ }^{2,3}$, \\ Myriam Gominet ${ }^{6}$, Jingni Yang ${ }^{1}$, Hengliang Wang7 , Qi Peng ${ }^{1}$, Jie Zhang ${ }^{1}$, Didier Lereclus ${ }^{2,3}$ \\ and Fuping Song ${ }^{1}$ \\ ${ }^{1}$ State Key Laboratory for Biology of Plant Diseases and Insect Pests, Institute of Plant Protection, Chinese \\ Academy of Agricultural Sciences, Beijing, China: ${ }^{2}$ INRA, UMR1319 Micalis, La Minière, 78280 Guyancourt, \\ France; ${ }^{3}$ AgroParisTech, UMR Micalis, F-78352 Jouy-en-Josas, France; ${ }^{4}$ Department of Life Sciences, Silwood \\ Park Campus, Imperial College London, Ascot SL57PY, UK; ${ }^{5}$ CAS Key Laboratory of Genome Sciences and \\ Information, Beijing Institute of Genomics, Chinese Academy of Sciences, Beijing, China; ${ }^{6}$ Institut Pasteur, \\ CNRS URA 2172, Unité de Biologie des Bactéries Pathogènes à Gram positif, Paris, France and ${ }^{7}$ Beijing \\ Institute of Biotechnology, State Key Laboratory of Pathogen and Biosecurity, Beijing, China
}

\begin{abstract}
A major challenge in bacterial developmental biology has been to understand the mechanisms underlying cell fate decisions. Some differentiated cell types display cooperative behaviour. Cooperation is one of the greatest mysteries of evolutionary biology and microbes have been considered as an excellent system for experimentally testing evolution theories. Bacillus thuringiensis $(\mathrm{Bt})$ is a spore-forming bacterium, which is genetically closely related to $B$. anthracis, the agent of anthrax, and to $B$. cereus, an opportunistic human pathogen. The defining feature that distinguishes $\mathrm{Bt}$ from its relatives is its ability to produce crystal inclusions in the sporulating cells. These toxins are solubilized after ingestion and are cooperative public goods in insect hosts. In this study, we describe a Bt strain LM1212 that presents the unique ability to terminally differentiate into crystal producers and spore formers. Transcriptional analysis based on lacZ and gfp reporter genes suggested that this phenotype is the consequence of a new type of cell differentiation associated with a novel regulation mode of cry gene expression. The differentiating crystal-producer phenotype has higher spore productivity than a typical Bt strain and is better able to compete with Cry toxin null 'cheaters'. Potentially, this division of labour provides additional fitness benefits in terms of spore viability or durability of Cry toxin.

The ISME Journal (2015) 9, 286-296; doi:10.1038/ismej.2014.122; published online 1 August 2014
\end{abstract}

\section{Introduction}

A major challenge in bacterial developmental biology is to understand how the bacteria determine their fate at the cell level (Losick and Desplan, 2008; Lopez et al., 2009). To date, this question has been addressed for several genera such as Streptomyces (Flardh and Buttner, 2009), Myхососcus (Kaiser et al., 2010), Caulobacter (Muro-Pastor and Hess, 2012) and Bacillus (Lopez et al., 2009). The model Gram-positive bacterium Bacillus subtilis can differentiate into several distinct cell types, which are controlled by a complex transcriptional regulatory network and are influenced by various extracellular signals (Lopez et al., 2009; Lopez and Kolter, 2010;

Correspondence: D Lereclus, INRA, UMR1319 Micalis, 78352 Jouy-en-Josas, France or F Song, The State Key laboratory for Biology of Plant Diseases and Insect Pests, Institute of Plant Protection, Chinese Academy of Agricultural Sciences, No.2 West YuanMingYuan Road, Beijing 100193, China.

E-mail: Didier.Lereclus@jouy.inra.fr or fpsong@ippcaas.cn

Received 9 February 2014; revised 12 May 2014; accepted 11 June 2014; published online 1 August 2014
Shank and Kolter, 2011). The production of differentiated cells is of great interest to evolutionary biologists as it is a critical step in the evolution of complex higher organisms (Buss, 1987; Maynard Smith and Szathmáry, 1995; Michod, 1997). Multicellular organisms, at their simplest, are collections of identical cells. When these groups are formed of genetically identical clones, they do not compete for reproduction (Buss, 1987). The absence of genetic conflict should pave the way for division of labour and the emergence of differentiated organisms.

Without doubt, multicellular organisms rely on cooperation, and can be thought of as aggregations of cooperating cells (Michod, 1999). Genetic differentiation via mutation can lead to conflict between cells and many important genetic processes (fair meiosis, chromosomes, the uniparental inheritance of organelles) may have arisen as a way of minimizing conflict (Maynard Smith and Szathmáry, 1995; Michod, 1997). The early evolution of differentiation is still not fully understood and various selection forces may have had important roles in different organisms. Differentiation may arise because of gains 
in productivity associated with division of labour (Maynard Smith and Szathmáry, 1995), because of a quantitative benefit associated with the production of somatic cells (Willensdorfer, 2009) or because incompatible physiological processes occur in different cell types. This incompatibility might be driven by tradeoffs in reproduction and motility (Michod and Roze, 2001) or through biochemical conflicts such as that between photosynthesis and oxygen-sensitive nitrogen fixation in cyanobacteria (Rossetti et al., 2010).

$B$. thuringiensis (Bt) is a spore-forming, Grampositive bacterium that has been studied for a century and successfully used as a biopesticide for more than 50 years (Bravo et al., 2011; Sanahuja et al., 2011). Bt is genetically closely related to $B$. anthracis, the agent of anthrax, and to B. cereus, an opportunistic human pathogen that causes foodborne gastroenteritis (Vilas-Boas et al., 2007). The defining feature that distinguishes $\mathrm{Bt}$ from its relatives is its ability to produce crystal inclusions in sporulating cells (Vilas-Boas et al., 2007). These crystals mainly consist of Cry and Cyt proteins that are generally toxic for specific insects (Bravo et al., 2011). As of January 2014, more than 700 Cry toxin genes organized in 72 classes had been discovered (http://www.lifesci.sussex.ac.uk/home/Neil_Crick more/Bt/). Only two mechanisms of transcriptional regulation of cry genes have been described, despite the large number of cry genes that have been identified: the binding of sporulation-specific sigma factors SigE and SigK to the promoters of cry genes, such as cry1, cry 4 or cry8, controls the transcriptional activity of these genes (Yoshisue et al., 1995; Du et al., 2012; Yang et al., 2012); the promoter of cry3 is sporulation independent and seems to be under the control of the main vegetative sigma factor SigA (Agaisse and Lereclus, 1994a,b). Nevertheless, all known Bt strains produce the crystal during spore formation within the same mother cell.

In common with many microbes, $B$. thuringiensis cooperates by secreting public goods, common shared resources that benefit local populations but that are costly for individuals to produce (Griffin et al., 2004; West et al., 2006). In Bt, the critical public good is solubilized Cry toxin in the insect midgut (Raymond et al., 2012). However, public goods' cooperation is particularly susceptible to competition from cheaters, non-producing strains that can exploit the products of cooperators but gain a growth advantage by avoiding the costs of production themselves (Griffin et al., 2004; Sandoz et al., 2007; West et al., 2007; Raymond et al., 2012). Although population structuring, or high relatedness, may reduce competition with Cry toxin cheaters (Raymond et al., 2012), this social conflict is likely to be a substantial selective force. Indeed, genetically diverse Cry toxin null strains in the $B$. cereus group can compete with toxin producers in a range of invertebrate hosts (Raymond et al., 2007; Kho et al., 2011).
In this study, we report a Bt strain, LM1212, with a unique phenotype: the formation of crystal does not occur in sporulating cells (called spore formers), but in a subpopulation of non-sporulating cells (called crystal producers) that lyse following production of Cry toxin inclusion bodies. Such a property has never been described in $\mathrm{Bt}$, despite the considerable number of Bt strains identified during the past few decades. Moreover, as Cry production is typically associated with sporulation, these two processes are not fundamentally incompatible. We suggest that a novel regulatory mechanism is responsible for the production of the Cry proteins, exclusively in the subpopulation of non-sporulating cells. These toxinproducing cells are expected to be more competitive in genetically diverse mixed infections or in competition with cheaters. Indeed, the LM1212 population could readily outcompete a Bt strain with a typical profile of crystal toxin production and could outcompete a Cry null strain, when it invaded an LM1212 population at low frequency.

\section{Materials and methods}

Strains, plasmids and culture conditions

The bacterial strains and plasmids used in this study are listed in Supplementary Table S1. Escherichia coli was incubated at $37^{\circ} \mathrm{C}$ in Luria-Bertani medium $(1 \%$ $\mathrm{NaCl}, 1 \%$ tryptone, $0.5 \%$ yeast extract). Bt was cultured at $30^{\circ} \mathrm{C}$ in sporulation medium SSM $(0.8 \%$ Nutrient broth, $0.1 \% \mathrm{KCl}, 0.12 \% \mathrm{MgSO}_{4}, 0.01 \mathrm{M} \mathrm{NaOH}, 0.01 \mathrm{M}$ $\mathrm{Ca}\left(\mathrm{NO}_{3}\right)_{2}, 0.1 \mathrm{~mm} \mathrm{MnCl}_{2}, 0.01 \mathrm{~mm} \mathrm{FeSO}_{4}$ ) (Schaeffer et al., 1965) or HCT (0.7\% casein hydrolysate, $0.5 \%$ tryptone, $\quad 0.68 \% \quad \mathrm{KH}_{2} \mathrm{PO}_{4}, \quad 0.012 \% \quad \mathrm{MgSO}_{4} \cdot 7 \mathrm{H}_{2} \mathrm{O}$, $0.00022 \% \quad \mathrm{MnSO}_{4} \cdot 4 \mathrm{H}_{2} \mathrm{O}, \quad 0.0014 \% \quad \mathrm{ZnSO}_{4} \cdot 7 \mathrm{H}_{2} \mathrm{O}$, $0.008 \%$ ferric ammonium citrate, $0.018 \% \mathrm{CaCl}_{2} \cdot 4 \mathrm{H}_{2} \mathrm{O}$, $0.3 \%$ glucose, $\mathrm{pH}$ 7.2) (Lecadet et al., 1980), with rotary agitation of 200 r.p.m. Ampicillin $\left(100 \mu \mathrm{g} \mathrm{ml}^{-1}\right)$ for E. coli or $5 \mu \mathrm{g} \mathrm{ml}^{-1}$ erythromycin for Bt were used to select the strains harbouring $\mathrm{pHT}$ plamids.

\section{DNA manipulation and transformation}

DNA manipulation (plasmid DNA extraction, DNA purification, digestion and ligation) is described in Supplementary Text S1. Oligonucleotide primers (Supplementary Table S2) were synthesized by Sigma-Proligo (Paris, France) and all constructs were confirmed by sequencing (Beckman Coulter Genomics, Takeley, UK). The transformation of E. coli was performed by a standard chemical-competent method and $B$. thuringiensis was transformed by electroporation as described previously (Lereclus et al., 1989).

\section{Microscopy}

Strains were cultured in appropriate medium and were collected at the indicated time points. Optical microscopy, transmission electron microscopy and confocal microscopy are described in Supplementary Text S2. 
Biochemical and microbiological phenotypic characterization

Haemolysis, ampicillin resistance and motility assays of Bt strains were tested on a blood plate (Columbia agar $+5 \%$ sheep blood, BioMérieux, Lyon, France), an ampicillin plate (Luria-Bertani with $1.3 \%$ agar $+100 \mu \mathrm{g} \mathrm{ml}^{-1}$ ampicillin) or a semisolid plate (Luria-Bertani $+0.3 \%$ agar), respectively, as described in Supplementary Text S3.

\section{4-sequencing and genomic analysis}

Chromosomal DNA from Bt LM1212 was prepared as described previously (Stobdan et al., 2004) and then sequenced by a 454 GS FLX platform (454 Life Sciences Corp., Roche, Branford, CT, USA). 16s rRNA typing, multiple locus sequence typing and delta-endotoxin genes exploring are described in Supplementary Text S4.

Crystal purification and peptide mass spectrometry identification

Crystal inclusions were purified using a $72-79 \%$ sucrose gradient and then analysed by SDS-polyacrylamide gel electrophoresis followed by matrix-assisted laser desorption/ionizationtime of flight mass spectrometry as described in Supplementary Text S5.

\section{Analysis of LM1212 cry mRNA}

Extraction of total RNA from Bt LM1212 (SSM medium, T0, T7, T13), cDNA synthesis and the reverse-transcription PCR analysis of cry open reading frames was performed as previously described (Du et al., 2012). The primers used in reversetranscription PCR are listed in Supplementary Table S2. Reverse transcriptase was omitted as a negative control. The transcription start sites of cry32Wa1 and cry35-like operons were determined by primer extension as previously described (Agaisse and Lereclus, 1996), using T7 RNA polymerase. Oligonucleotides complementary to the downstream region of these promoters (p32-PE for cry32Wa1 and p35-PE for cry35-like) were used.

\section{Bioassays}

Details for bioassay are described in Supplementary Text S6.

For mammal infection assay, mice were treated with hypodermic injection of vegetative cells or with intragastric administration of spores.

The assays for the insect larva Plutella xylostella, Colaphellus bowringi, Agrotis ypsilon, Spodoptera exigua and Aedes aegypti were performed using crystal-spore mixture or purified crystals of LM1212.

For cytotoxicity assay, purified, protease-activated LM1212 crystals were tested in six cancer cell lines (Caco-2, HL60, Jurkat, HepG2, Hela and MOLT4).
Crystals from HD73 $\mathrm{Cry}^{-}\left(\mathrm{p}^{-} \mathrm{E}^{\prime}\right.$ cry41Ca) and HD73 Cry $^{-}\left(\mathrm{p} 8 \mathrm{E}^{\prime}\right.$ cry $\left.45 \mathrm{Ba}\right)$ were purified and their cytotoxicity was tested in two cell lines (Caco-2 and HeLa) after protease activation.

\section{Plasmid construction}

Plasmids were constructed by PCR methods. Details are described in Supplementary Text S7.

\section{$\beta$-galactosidase activity assay}

Samples $(2 \mathrm{ml})$ of cells cultured in SSM medium with antibiotics were collected at the indicated time points. The activity was measured as previously described and expressed as units per milligram of protein (Perchat et al., 2011). Each assay was repeated three times.

\section{Competition experiment}

Competition experiments were performed between $\mathrm{LM}\left(\mathrm{p} 35^{\prime} \mathrm{Z}\right)$ and $\mathrm{HD} 73\left(\mathrm{p} 35^{\prime} \mathrm{Z}\right)$ or $\mathrm{LM}\left(\mathrm{p} 35^{\prime} \mathrm{Z}\right)$ and HD73Cry $^{-}\left(\mathrm{p}^{\prime} 5^{\prime} \mathrm{Z}\right)$. Cells were co-cultured in HCT medium at different initial cell ratios with a temperature of $30^{\circ} \mathrm{C}$, until sporulation was complete. Spores were heat treated and plated on X-gal dishes as described in Supplementary Text S8. The experiment was repeated five times.

Data were analysed in R 3.0.2 (The R Foundation for Statistical Computing, http://www.R-project.org) using generalized linear modelling approaches, assumptions of normality and homoscedasticity were checked with graphical analyses. We used the change in the proportion of HD73 strains as measure of relative fitness ( $V$ ), following RossGillespie et al. (2007). Analysis of fitness used natural log-transformed relative fitness $(V)$ as a response variable.

\section{Results}

Phenotypic and genetic characterization of LM1212 Spores of the LM1212 strain were isolated from the cadaver of a rhinoceros beetle larva Oryctes gigas in Madagascar more than 10 years ago (Chaufaux et al., 1997). This strain was singled out from another 1000 isolations because of its unique and intriguing differentiation phenotype: about $60 \%$ of the population presented a spore without any inclusion, whereas about $40 \%$ was not sporulating but contained a large, round inclusion (Figures 1a and b). Transmission electron microscopy revealed that the inclusions were crystals (Figure 1C), suggesting that LM1212 was probably a strain of Bt. However, this is different from all other identified Bt strains, which form crystal and spore in the same mother cell (Supplementary Figure S1). Phenotypic analysis showed that LM1212 was haemolytic on sheep blood agar and was resistant to ampicillin (Supplementary Figures S2A and B). These phenotypes 


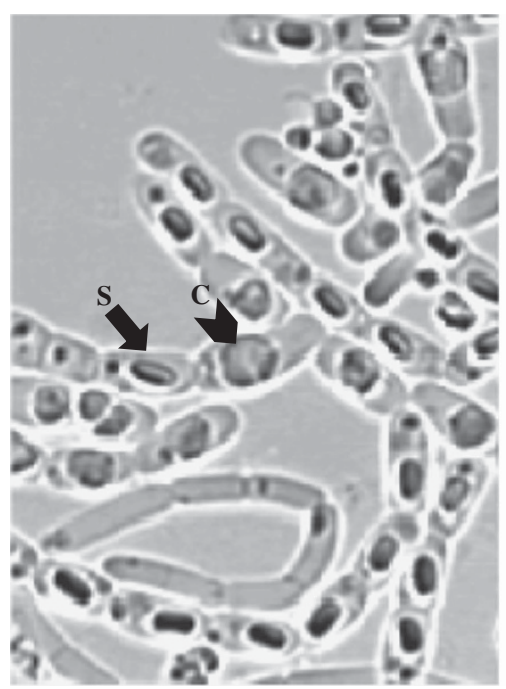

b

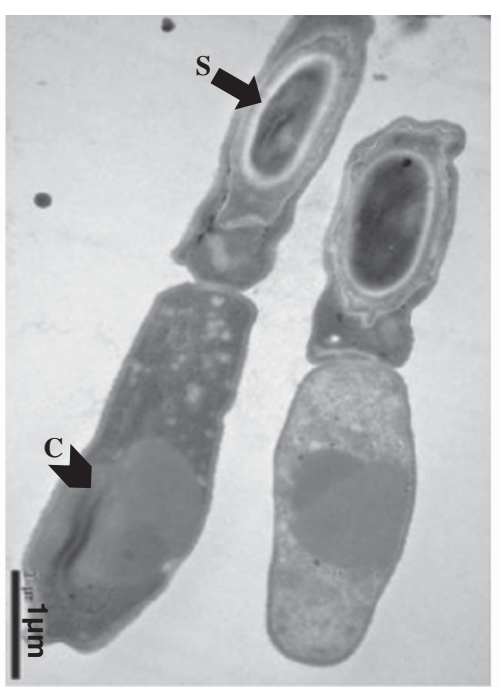

C

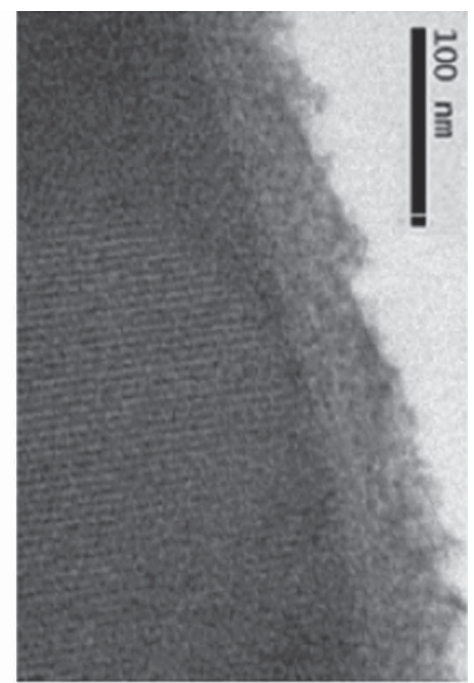

Figure 1 Differentiation of crystal producers and spore formers in LM1212. The spores and crystals appear in distinct cells in LM1212. Pictures of (a) optical micrograph and (b) transmission electron microscopy (TEM). (c) TEM reveals the crystal lattice in LM1212 inclusions. S, spore; C, crystal toxin.

are observed for most Bt strains (Vilas-Boas et al., 2007). However, in contrast with most Bt strains (Vilas-Boas et al., 2007), LM1212 was non-motile (Supplementary Figures S2C and D). The bacteria also displayed a long-chain cell phenotype (Supplementary Figures S2E and F).

To characterize the LM1212 strain in more detail, we performed 454-sequencing and obtained a $6 \mathrm{Mbp}$ draft genome with 52 scaffolds (accession number: AYPV00000000). Phylogenetic analysis of the 16s RNA gene showed that LM1212 was most closely related to the Bt serovar konkukian strain 97-27 (Supplementary Figure S3A), a strain that was originally isolated from a case of human tissue necrosis and that could infect and kill immunosuppressed mice (Hernandez et al., 1998). The 16s rRNA of LM1212 did not belong to any of the 16S rRNA types defined by Sacchi et al. (2002), but resembled closely type 10 of Bt (Supplementary Table S3). Multiple locus sequence typing analysis showed that LM1212 was most closely related to the Bt serovar finitimus strain YBT-020 (Supplementary Figure S3B), a strain that displays a 'spore-crystal association' phenotype in that it produces crystals inside the exosporium (Zhu et al., 2011). These genomic analyses confirm that LM1212 is a Bt strain. Moreover, the LM1212 genome contains genes for PlcR and NprR, which are quorum-sensing regulators specific to the $B$. cereus group bacteria (Slamti and Lereclus, 2005; Perchat et al., 2011). The sequences of the sensor regulators (PlcR and NprR) and of their cognate peptides (PapR and NprX) from LM1212 were $100 \%$ identical to those of $B$. cereus G9241 (data not shown), a strain encoding the entire anthrax toxin biosynthetic complex and that was responsible for a human anthrax case (Hoffmaster et al., 2004). This is surprising because PlcR-PapR and NprR-NprX are phylogenetically unrelated and these two systems are supposed to have evolved independently (Perchat et al., 2011). Thus, like its close relative B. cereus G9241, LM1212 may be a potential mammalian pathogen. The LM1212 genome did not contain the $B$. anthracis toxin-related genes $\operatorname{pag} A, \operatorname{cya}, \operatorname{cap} A / B / C / D$ and $a t x A$. In agreement with the absence of the cap genes, LM1212 did not produce capsule detectable by Indian ink staining (data not shown). However, LM1212 possessed a hypothetical protein displaying a high similarity ( $42 \%$ identity, $64 \%$ positive) to chain A of lethal factor (Supplementary Figure S4F). We assessed the toxicity of the LM1212 strain in mice by hypodermic injection of vegetative cells and intragastric administration of spores, using the $B$. anthracis vaccine strain A16R (Liu et al., 2013) as positive control, and showed that LM1212 did not infect mammals (Supplementary Table S4).

Characterization and expression of the LM1212 crystal proteins and reverse-transcription PCR analysis of cry gene transcription in LM1212

A BLAST search of an in-house Bt toxin database using the LM1212 genome as input revealed seven putative cry genes (cry32Wa1, cry32Va1, cry35-like, cry35-like2, cry41Ca1, cry45Ba1 and cry74Aa1) and two putative $c y t$ genes (cyt1Aa-like and cyt1Ca-like; Supplementary Table S5). We purified LM1212 crystals by sucrose gradient centrifugation and analysed them by SDS-polyacrylamide gel electrophoresis followed by matrix-assisted laser desorption/ionization-time of flight mass spectrometry. All seven full-length Cry-like proteins and two other proteins (a NT32-like protein and a putative protein) were identified as components of LM1212 crystals 


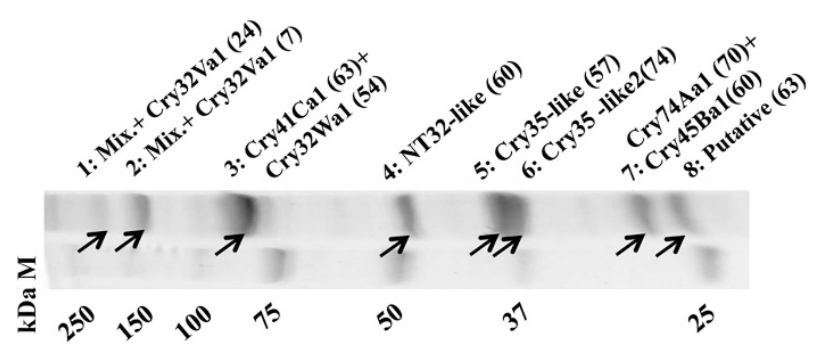

Figure 2 SDS-polyacrylamide gel electrophoresis (PAGE) and mass spectrometry (MS) analyses of LM1212 crystals. Purified LM1212 crystals were analysed by 10\% SDS-PAGE. Eight bands (1-8, indicated by arrows) were selected for identification by matrix-assisted laser desorption/ionization-time of flight MS. All seven expected Cry-like proteins but no Cyt-like protein were detected. In addition, a NT32-like protein (4) and a putative protein (8) were found as components of LM1212 crystals. $\mathrm{M}$, protein standard; Mix, mixture of truncated Cry-like proteins. Numbers in parentheses show peptide coverage (\%) in MS analysis.

(Figure 2). However, no Cyt-like protein was detected in crystals (Figure 2). On the basis of the amino-acid sequences, five delta endotoxins in LM1212 (Cry32Wa1, Cry32Va1, Cry41Ca1, Cry45Ba1 and Cry74Aa1) were classified as novel class I Cry toxins (Supplementary Table S5) and four Cry proteins (Cry32Wa1, Cry41Ca1, Cry45Ba1 and Cry74Aa1) showed a high degree of similarity to the cancer cell-killing protein parasporins (Ohba et al., 2009; Supplementary Table S5; Supplementary Figures S4A and B). The two Cry35-like proteins were similar to the Cry35 toxin of Bt (Schnepf et al., 2005; Supplementary Figure S4C) and to the binary toxins BinA and BinB of Lysinibacillus sphaericus (formerly known as B. sphaericus; Berry, 2012; Supplementary Figures S4D and E).

Four operons containing the binary toxin-like and parasporin-like genes were successfully cloned and expressed in the Bt HD73 crystal minus strain (HD73 Cry $^{-}$; Supplementary Figures S5 and S6). However, we found that LM1212 crystal proteins had no significant toxicity to a range of cancer cell lines or insects (Supplementary Tables S6 and S7).

Activity of the LM1212 cry gene promoters is restricted to a specific subpopulation of the bacterial cells

We selected the cry32Wa1 and cry35-like operons, which encode the major Cry proteins of the LM1212 crystals (Figure 2), to investigate transcriptional regulation of the LM1212 cry genes. Primer extension revealed that the transcription start sites of cry32Wa1 operon were 34 and 35 nucleotides (nt) upstream from the putative translation start of the p19-like open reading frame (Figure 3a; Supplementary Figure S5A). The transcription start sites of the cry35-like operon mapped to 36 and $37 \mathrm{nt}$ upstream from the putative start codon of the cry35like gene (Figure 3c; Supplementary Figure S5B). The -10 region of the two promoters resembles that recognized by the vegetative sigma A factor (Moran et al., 1982). In sharp contrast, the -35 regions did not fit the consensus region recognized by this sigma factor (TTGCATA). Alignment of the -35 regions of the two promoters revealed a conserved DNA sequence (ATTTCCA $/ \mathrm{CTCC}_{/ \mathrm{A}} \mathrm{TCG}_{/ \mathrm{A}} \mathrm{GTT}$ ) that might be the target of a transcriptional regulator.

We cloned each of these promoter regions $\left(\mathrm{P}_{\text {cry32Wa1 }}\right.$ and $\mathrm{P}_{\text {cry35-like }}$ ) upstream of the reporter gene lac $Z$. We then transformed these plasmid constructs, which we named $\mathrm{p} 32^{\prime} \mathrm{Z}$ and $\mathrm{p} 35^{\prime} \mathrm{Z}$, into the Bt LM1212 and HD73 strains to generate LM(p32'Z), LM(p35'Z), HD73(p32'Z) and HD73(p35'Z). In LM1212, transcription of these two constructs was activated from the end of logarithmic growth (T0) and continually increased for several hours (Figures $3 \mathrm{~b}$ and $\mathrm{d}) . \mathrm{P}_{\text {cry35-like }}$ appeared to be stronger than $\mathrm{P}_{\text {cry32Wa1 }}$ (Figures $3 \mathrm{~b}$ and $\mathrm{d}$ ), which is consistent with the profile of these proteins on SDS-polyacrylamide gel electrophoresis (Figure 2). In contrast, the activity of these two promoters was very low in the HD73 strain throughout the growth (Figures 3b and $\mathrm{d}$ ). These results suggest that the transcription of these cry genes might depend on transcription factors specific to the LM1212 strain. As the production of crystal was restricted to non-sporulating cells of LM1212 (Figure 1b), a second hypothesis would be that the LM1212 cry genes were transcribed only in the subpopulation of non-sporulating cells in both LM1212 and HD73. This subpopulation may have been smaller in HD73 than in LM1212.

To test these hypotheses, we cloned either $\mathrm{P}_{\text {cry32Wa1 }}$ or $\mathrm{P}_{\text {cry35-like }}$ upstream of the reporter gene $g f p$, and transformed the LM1212 and HD73 strains with these plasmid constructs (named p32'gfp and p35'gfp). We monitored growth and fluorescence of the resulting strains $\mathrm{LM}\left(\mathrm{p} 32^{\prime} g f p\right), \mathrm{LM}\left(\mathrm{p} 35^{\prime} g f p\right)$, HD73(p32'gfp) and HD73(p35'gfp) using a confocal microscope. As expected, in LM1212, fluorescence was restricted to the subpopulation of crystal producers and absent in the spore formers (Figures 4a and b; Supplementary Figures S7A and B). The expression of $g f p$ was low in a few cells at the end of logarithmic growth (T0), and the fluorescence intensity increased from T4 to T20 (Supplementary Figures S7A and B). In agreement with the $\beta$-galactosidase assays (Figures $3 \mathrm{~b}$ and $\mathrm{d}$ ), the $\mathrm{LM}\left(\mathrm{p} 35^{\prime} g f p\right)$ strain presented a higher fluorescence intensity than LM(p32'gfp). In HD73, the cry promoters were transcribed only in the small subpopulation of non-sporulating cells, although at a much lower intensity than in LM1212 (Figures 4c and d; Supplementary Figures S7C and D), implying the effect of potential transcriptional regulator(s) specific to the LM1212 strain. We did not detect green fluorescent protein fluorescence in sporulating cells (Figures 4c and d; Supplementary Figures S7C and D).

To investigate the transcription pattern of other cry gene promoters in LM1212 strain, we cloned a typical sporulation-dependent cry gene promoter 
a

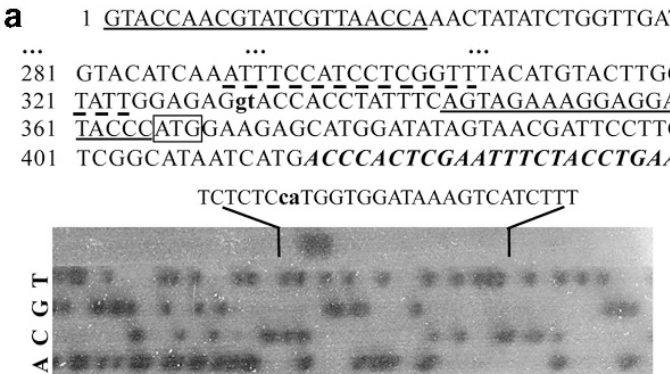

b

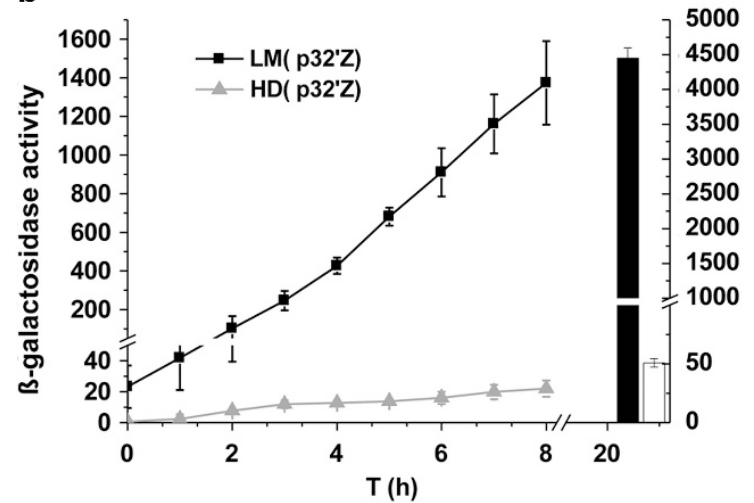

C

CACCTTTTAaAgTagTagTaCCAGTATGGCCAAATTTGAa

161 AAATAAGCACAATATTTCCCTCATCAGTTCATATGTCATT 201 GCTAGACTGAAGatGTATTATA ÄÄATTAĀTCCGAAAGGGG 241 AACTGTTTGATGCCTACAATAGAAAAAGGGCGACCTTATA 281 AAATT GCTTTAAGGGCAAATCGAAATGTGGTA

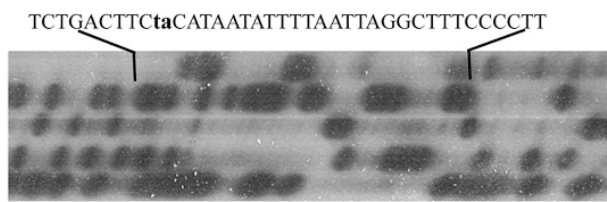

d

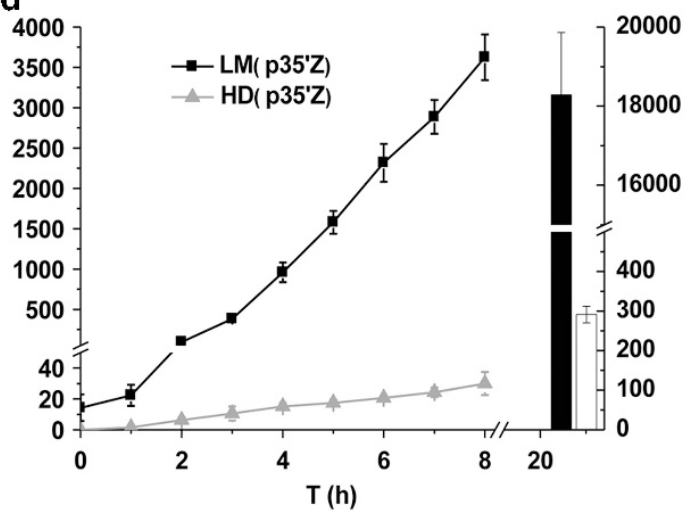

Figure 3 Transcriptional analysis of LM1212 cry genes. (a and c) Sequence and primer extension analyses of cry32Wa1 and cry35-like promoters. Primers used for cloning the promoter regions are underlined and primers for extension experiments are indicated by black italics. Putative translation start codons are boxed and transcription start sites (TSSs) are noted with bold lowercases. The putative - 10 and -35 regions of the promoters are indicated by dashed lines. The sequence extension results are noted above the gels and TSSs are shown with high black lowercases. (b and d) The activity of cry32Wa1 and cry35-like promoters as assessed by lacZ fusions. The columns on the right of the diagrams indicate the $\beta$-galactosidase activity of T20 (LM1212: black columns and HD73: white columns). Data are an average of three independent assays. Error bar is the s.d. Cells were cultured in SSM medium. T0 is the end of logarithmic growth and Tn means $n$ hours after T0.

$\mathrm{P}_{\text {cry1Ac }}$, which is under the control of SigE and SigK (Yang et al., 2012), from the HD73 strain upstream of a $g f p$ reporter gene to create the plasmid construct p1Ac'gfp. In contrast to the LM1212 cry gene promoters, the expression of this construct was restricted to sporulating cells and was not observed in the population of non-sporulating cells in both LM1212 and HD73 strains (Figures 4e and f; Supplementary Figures S7E and F). As controls, we tested a $g f p$-fusion construct of the constitutive promoter of the $a p h A 3$ gene $\left(\mathrm{p}_{\text {aphA3 }}\right)$, which was ubiquitously expressed (Supplementary Figure S7G), and the promoter-less plasmid pHT-R-l-gfp, which had a very low background expression (Supplementary Figure S7H). We transformed two additional Bt wild-type strains, Bt22 and YBT1520 (laboratory stock), the $B$. cereus type strain ATCC14579 (Bacillus Genetic Stock Center) and the B. subtilis strain 168 (Bacillus Genetic Stock Center) with p32'gfp. As observed for the HD73 strain (Figures 4c and d), a small subpopulation of non-sporulating cells expressed this fusion construction in these four Bacillus strains (Supplementary Figure S8).

All the above results indicate that the LM1212 cry promoters are controlled by a novel mode of transcriptional regulation that restricts their activity to a subpopulation of non-sporulating cells.
Productivity and competitive ability of LM1212

In the LM1212 strain, the crystal producers account for only about $40 \%$ of the final population, so we hypothesized that LM1212 would yield more spores than a competing Bt strain that produces crystals in all sporulating cells, as crystal toxin production has substantial metabolic costs (Agaisse and Lereclus, 1995; Raymond et al., 2012). We tested this hypothesis in single-strain spore yield experiments and in competition experiments between LM1212 and HD73. We co-cultured LM(p35'Z) and HD73(p35'Z) in sporulating medium with different initial cell ratios for $48 \mathrm{~h}$. We then heat treated spores and plated them on media containing X-gal. The activity of the $\mathrm{P}_{c r y 35 \text {-like }}$ promoter in the HD73 strain is very low (Figure 3d), meaning that the LM1212 (blue) and HD73 (white or very light blue) clones were easily distinguishable because LM1212 expressed a higher amount of LacZ than HD73 (Figure 3d).

As we predicted, spore productivity declines as the proportion of cells producing Cry toxin increases, so that the LM1212 has a spore yield that was intermediate between that of the Cry positive and the Cry negative HD73 strains (Figure 5A; $\left.F_{2,12}=4.69, P=0.0292\right)$. The competition experiments confirmed this result. When competition experiments were initiated with $<70 \%$ HD73 Cry positive bacteria, LM1212 showed higher fitness 
a

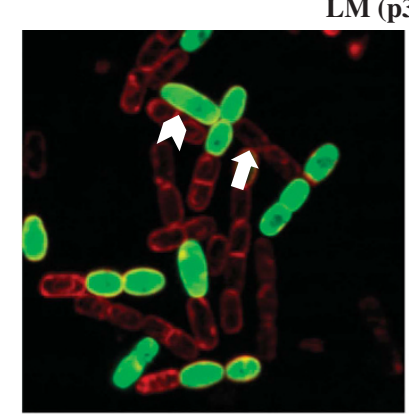

C

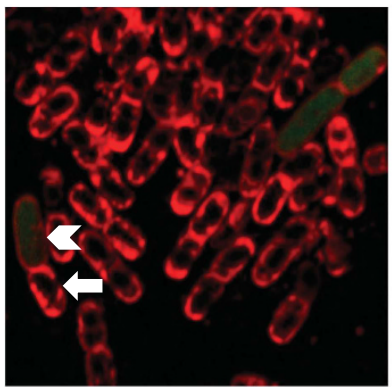

e

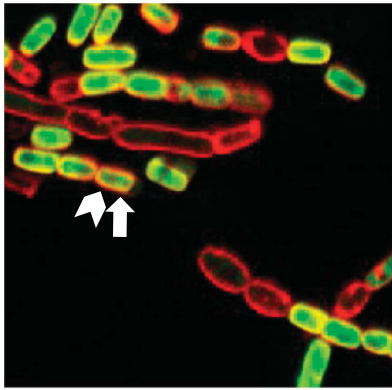

$\mathbf{L M}(\mathbf{p} 1 \mathrm{Ac} \cdot g f p)$

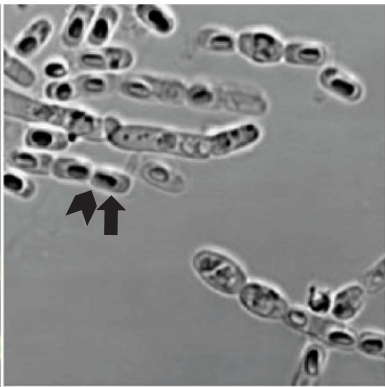

b

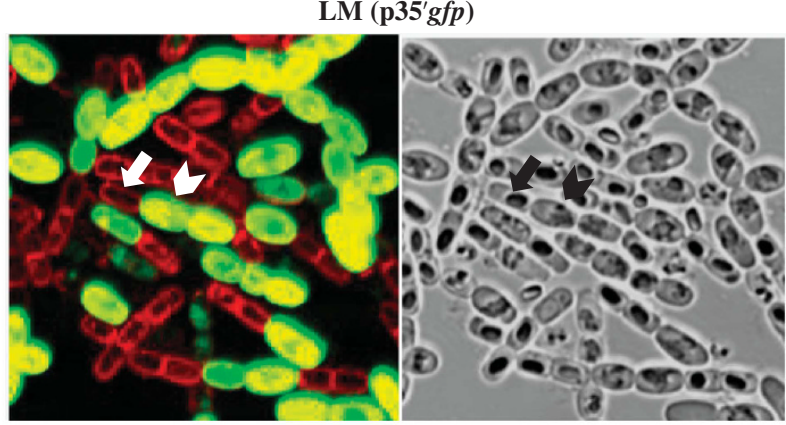

d

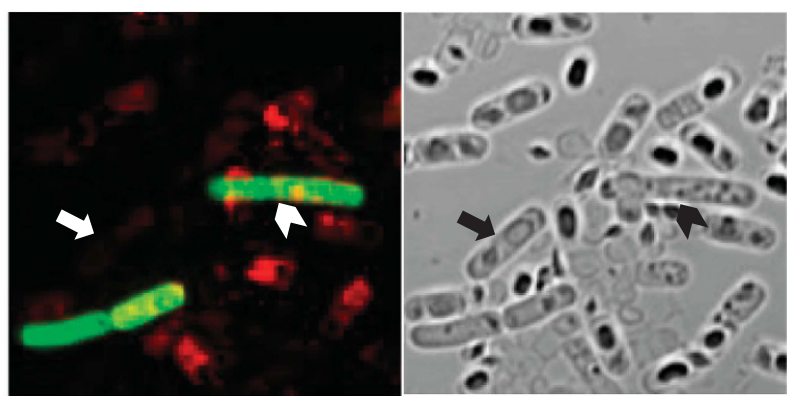

f

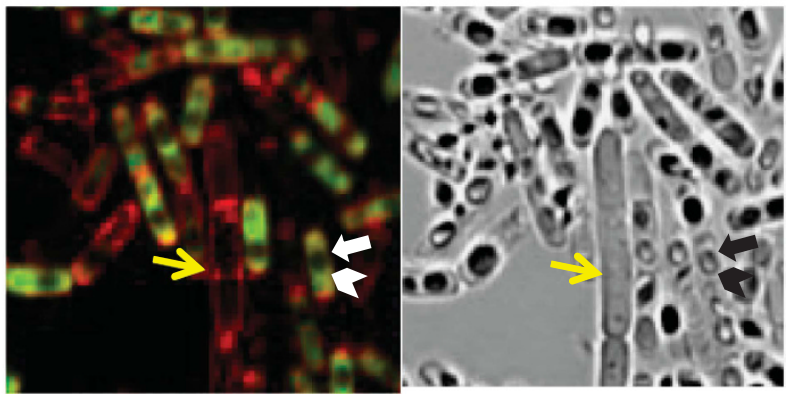

Figure 4 The activity of LM1212 cry promoters is restricted to non-sporulating cells in both LM1212 and HD73 strains. We used a gfp reporter gene to assess the activity of the P32 and P35 promoters in LM1212 (a and b) and in HD73 (c and d). The activity of the sporulation-dependent cry gene promoter $\mathrm{P}_{c r y 1 A c}$ is restricted to the sporulating cells in LM1212 and HD73 (e and f). Non-sporulating cells in HD73 are indicated by yellow arrows. Left: GFP/FM-4-64 overlay. Right: bright-field image. Sporulating cells are indicated by arrows and green fluorescent protein-expressing cells are indicated by arrow heads. Cells were grown in SSM medium until T20 with erythromycin. The cell walls were stained with FM-4-64.

than this conventional Bt strain (Figure 5B). Although we found no evidence of overt mutual antagonism between LM1212 and HD73 on agar plates (Supplementary Figure S9), the fitness of HD73 was positively frequency dependent in both competition experiments (Figures $5 \mathrm{~B}$ and $\mathrm{C}$; $\mathrm{F}_{2,12}=28.9, P=0.0292$ ), suggesting that there were some antagonistic interactions between these genetically distinct strains when they are co-cultured in broth. To check whether the overall fitness advantage of LM1212 to HD73 was due to the different levels of investment in Cry toxins, we carried out a similar competition experiment between LM1212 and the HD73 $\mathrm{Cry}^{-}$strain (transformed with p35'Z), which does not produce crystal toxin. In these conditions, LM1212 was generally outcompeted by HD73 Cry $^{-}$(Figure 5C). Importantly, this Cry null strain was not capable of outcompeting LM1212 at low initial frequencies (Figure 5C). The full analysis of relative fitness used strain as a main effect and initial proportion of HD73 as a covariate, fitted as both linear and quadratic terms. Model simplification showed that strain interacted significantly with initial proportion $\left(F_{1,43}=15.6, \quad P=0.00028\right)$ and initial proportion squared $\left(\mathrm{F}_{1,43}=18.3, P=0.0001\right.$; Figures 5B and C).

\section{Discussion}

The most intriguing feature of LM1212 is its ability to differentiate into two distinct subpopulations: crystal producers and spore formers (Figures 1a and b). This ability is determined by specialized promoters, which restrict expression of cry genes to nonsporulating cells, and implies the existence of a novel mode of transcriptional regulation. In Bt, the cost of the production of Cry toxin inclusions is 

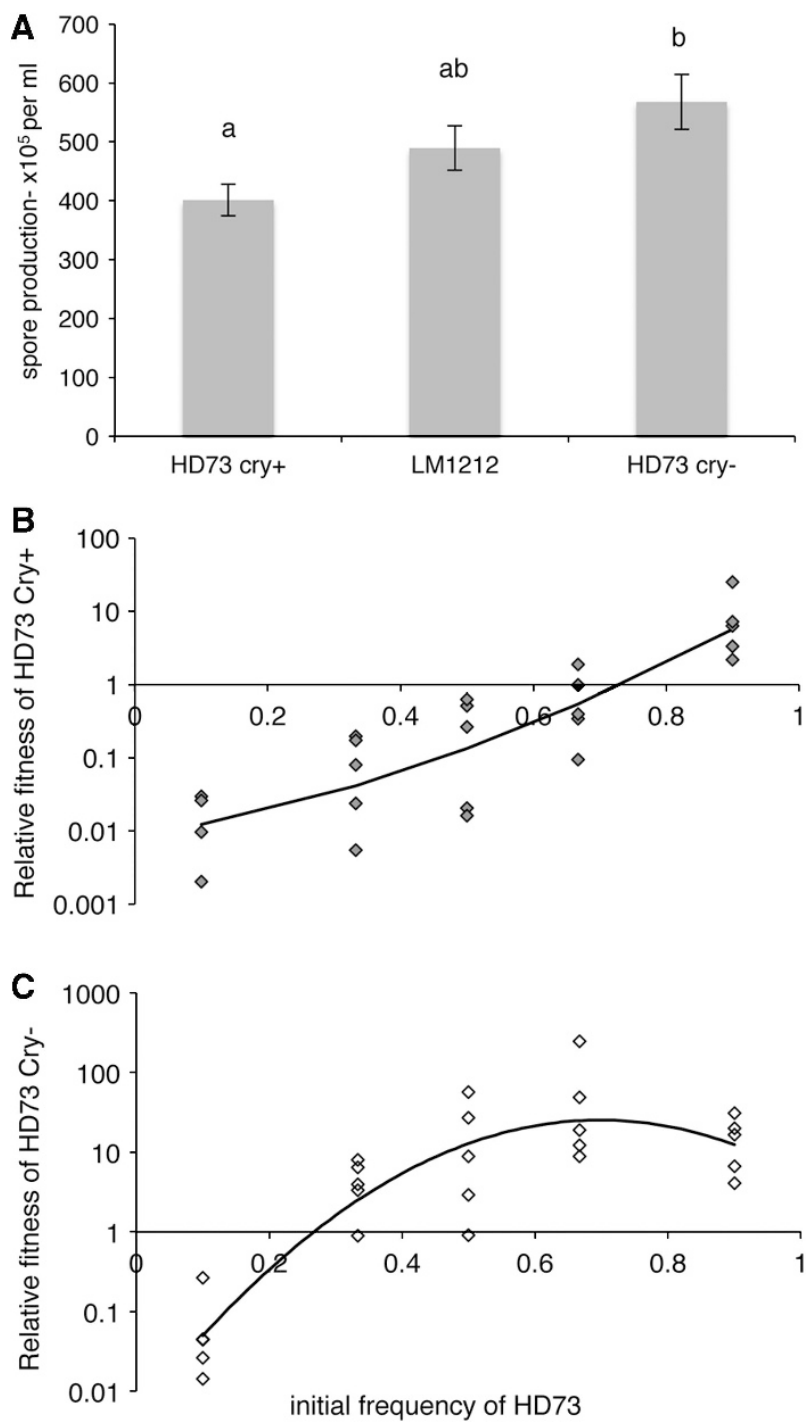

Figure 5 Productivity of LM1212 and relative fitness in competition with Bt strains. (A) The spore yield of LM1212 is intermediate between that of a canonical Bt strain and a cry null mutant, data are means \pm s.e. Different superscripts indicate significantly different means, according to post hoc treatment contrasts, after fitting generalized linear models. (B) LM(p35'Z) has a positive frequency-dependent fitness, but outcompetes HD73(p35'Z) over a wide range of initial conditions. (C) A Cry null strain, HD73Cry ${ }^{-}\left(\mathrm{p} 35^{\prime} \mathrm{Z}\right)$, can outcompete LM(p35'Z), except when rare. Spores were heat treated at $65^{\circ} \mathrm{C}$ for $25 \mathrm{~min}$ before plating. Lines are fitted statistical models.

substantial both in terms of pure mass ( $\sim 25 \%$ of the total dry weight of $\mathrm{Bt}$ ) and metabolism (Agaisse and Lereclus, 1995). Crystal toxins are considered as public goods because they can be exploited by cheaters that do not produce such proteins in the insect host (Agaisse and Lereclus, 1995; Raymond et al., 2012). In LM1212, the crystal producer makes a crystal that fills nearly half of the inflated cell (Figures 1a and b). This behaviour is energy consuming for an individual cell but presumably benefits the spore-forming members of the population in terms of facilitating pathogenic infections. Ultimately, these Cry toxin-producing cells lyse, so without making a spore, these cells have no direct way of passing on genetic material to the next generation.

The specialization of cell types into spore formers and toxin producers represents an altruistic division of labour. The evolution and development of specialized cell types has been of great interest to evolutionary biologists, as it represents an early and important step in the evolution of complex multicellular organisms (Bell, 1985; Maynard Smith and Szathmáry, 1995). Analogous division of labour occurs in other microorganisms, most notably in the division of germ and soma in Volvox and the production of non-reproductive 'stalks' in the fruiting bodies of bacteria and social amoeba (Bell, 1985; Strassmann et al., 2000; Velicer et al., 2000). A key prediction of the economic theory of the division of labour is that specialized subdivision of tasks should result in increased production efficiency (Maynard Smith and Szathmáry, 1995), a prediction that is often inferred rather demonstrated directly. Here we show that division of labour increases the spore yield of LM1212, at least in relation to a canonical Bt strain that produces Cry toxin in every sporulating cell. Cry toxin production is very costly, and invertebrate hosts represent a fixed pool of resource, so that mixed infection of Cry toxin producers and Cry null mutants can produce more viable spores per host than pure infections of Cry toxin producers (Raymond et al., 2012). Peak spore production of $\mathrm{Bt}$ in lepidopteran larvae occurs in infections initiated with between $40 \%$ and $80 \%$ toxin producers (Raymond et al., 2012). The fact that the crystal producers in LM1212 constitute $40 \%$ of the population suggests that this ratio has been under selection to maximize reproductive rates while retaining Cry toxin production.

However, a naive prediction is that similar gains in spore productivity could be achieved by simply reducing the amount of Cry toxin per cell, so we would predict that there must be some substantial fixed cost per cell of initiating the production of parasporal inclusions, perhaps in terms of reduced spore viability ( $\mathrm{Du}$ and Nickerson, 1996). Conversely, there may be a benefit in terms of Cry toxin durability by producing toxin in specialized cells, as the production of encapsulated Cry toxins in a sporulation-deficient $\mathrm{Bt}$ strain has been shown to protect the toxins from sunlight and UV damage (Sanchis et al., 1999).

An additional benefit of division of labour would be to reduce the average per cell synthesis of Cry toxin, so that genotypes with a subpopulationspecific expression of toxin should be more competitive in mixed infections with other members of the $B$. cereus group. Consistent with this prediction, we found that the competitive fitness of LM1212 in sporulation media was intermediate between that of a typical Cry toxin producer and a Cry null strain. LM1212 outcompeted Cry toxin producers over a wide range of initial frequencies. Critically, LM1212 
could even outcompete a Cry null strain when this competitor was invading from rare (Figure 5C). Previous competition experiments with Bt Cry toxin producers have shown that Cry null cheaters can very successfully invade from rare in field conditions (Raymond et al., 2012). Although the experiments in the study did not use isogenic mutants, this is probably ecologically realistic. Given the diversity of $B$. cereus group strains in local populations (Sorokin et al., 2006; Bizzarri et al., 2008; Raymond et al., 2010b), most competition that occurs between toxin producers and non-toxin producers in the field is likely to occur between genetically distinct strains.

The three most studied species of the $B$. cereus group, B. anthracis, B. thuringiensis and B. cereus, are genetically very closely related. $B$. anthracis and $\mathrm{Bt}$ are generally identified by their phenotypic traits that depend on the presence of particular plasmid genes. Notably, B. anthracis produces a capsule and anthrax toxins and Bt synthesizes entomopathogenic parasporal inclusions (Kolsto et al., 2002). In contrast, $B$. cereus does not present these distinctive traits. These species names do not represent good monophyletic groups, so it is difficult to distinguish them using chromosomal genetic typing methods (Helgason et al., 2000; Vilas-Boas et al., 2007; Raymond et al., 2010a). However, phylogenetic analysis of strains characterized by multiple locus sequence typing suggests that closely related strains are likely to have similar ecology or host association (Guinebretière et al., 2010; Raymond et al., 2010b; Raymond and Bonsall, 2013a). In this study, we identified LM1212 as a strain of Bt, based on both genetic typing methods (Supplementary Figure S3; Supplementary Table S3) and phenotypic identification (Figures 1b and 2; Supplementary Figures S2A and B). LM1212 proved to be closely related to Bt serovar konkukian strain 97-27 and to Bt serovar finitimus strain YBT-020. Both these strains cluster within the $B$. cereus group clade that contains $B$. anthracis, a clade variously described as 'group 1', 'group III', 'the anthrax clade' or the 'C clade' (Priest et al., 2004; Sorokin et al., 2006; Guinebretière et al., 2010; Raymond et al., 2010b). The PlcR-PapR and NprR-NprX systems (Perchat et al., 2011) of LM1212 were found identical to those of the anthrax-casing B. cereus G9241, although it contained no anthrax toxin genes and did not produce a capsule. Broadly, strains in the anthrax clade are more likely to be associated with vertebrate hosts, whereas most entomopathogenic Bt strains fall into a different clade (Raymond and Bonsall, 2013b). The natural host affiliations of Bt strains in the anthrax clade are almost entirely unknown and so it was not surprising that we were unable to identify an invertebrate host. In general, more than $90 \%$ of Bt isolations do not have a known target (Ohba et al., 2009).

In summary, we describe an unusual strain of B. thuringiensis, LM1212. This strain has a unique feature: the separation of crystal and spore-forming activities, a division of labour entirely unknown in B. thuringiensis. This intriguing phenotype is the consequence of a new type of cell differentiation associated with a novel form of regulation of cry gene expression. The toxin producer is lytic and presumably benefits its clone mates by producing energy-costly toxin inclusions and pays a cost in terms of being a 'sterile worker'. Suicidal, lytic secretion of public goods has previously been identified in colicin producers in E. coli (Chao and Levin, 1981) but is generally rare in microbes. This division of labour in the LM1212 population appears to increase its spore productivity and competitive ability by reducing average levels of toxin synthesis. However, we are yet to elucidate the benefits of concentrating Cry toxin production in a specialized group of cells, rather than reducing mean levels of production in all cells.

\section{Conflict of Interest}

The authors declare no conflict of interest.

\section{Acknowledgements}

We thank Céline Henry for the mass spectrometry analysis (INRA, Micalis, PAPSSO platform). This work was supported by a grant from the National Natural Science Foundation of China (No. 31270111) and by the French National Institute of Agronomical Research.

\section{References}

Agaisse H, Lereclus D. (1994a). Expression in Bacillus subtilis of the Bacillus thuringiensis cryIIIA toxin gene is not dependent on a sporulation-specific sigma factor and is increased in a spooA mutant. J Bacteriol 176: $4734-4741$.

Agaisse H, Lereclus D. (1994b). Structural and functional analysis of the promoter region involved in full expression of the cryIIIA toxin gene of Bacillus thuringiensis. Mol Microbiol 13: 97-107.

Agaisse H, Lereclus D. (1995). How does Bacillus thuringiensis produce so much insecticidal crystal protein? J Bacteriol 177: 6027-6032.

Agaisse H, Lereclus D. (1996). STAB-SD: a Shine-Dalgarno sequence in the $5^{\prime}$ untranslated region is a determinant of mRNA stability. Mol Microbiol 20: 633-643.

Bell G. (1985). The origin and early evolution of germ cells as illustrated by the Volvocales. In: Halvorson $\mathrm{H}$, Mornoy A (eds) The Origin and Evolution of Sex. Alan R. Liss: New York, NY, USA, pp 221-256.

Berry C. (2012). The bacterium, Lysinibacillus sphaericus, as an insect pathogen. J Invertebr Pathol 109: 1-10.

Bizzarri M, Prabhakar A, Bishop A. (2008). Multiple-locus sequence typing analysis of Bacillus thuringiensis recovered from the phylloplane of clover (Trifolium hybridum) in vegetative form. Microb Ecol 55: 619-625.

Bravo A, Likitvivatanavong S, Gill SS, Soberon M. (2011). Bacillus thuringiensis: a story of a successful bioinsecticide. Insect Biochem Mol Biol 41: 423-431. 
Buss LW. (1987). The Evolution of Individuality. Princeton University Press: Princeton, NJ, USA.

Chao L, Levin BR. (1981). Structured habitats and the evolution of anticompetitor toxins in bacteria. Proc Natl Acad Sci USA 78: 6324-6328.

Chaufaux J, Marchal M, Gilois N, Jehanno I, Buisson C. (1997). Reasearch on natural strains of Bacillus thuringiensis in different biotopes throughout the world. Can J Microbiol 43: 337-343.

Du C, Nickerson KW. (1996). Bacillus thuringiensis HD-73 spores have surface-localized Cry1Ac toxin: physiological and pathogenic consequences. Appl Environ Microbiol 62: 3722-3726.

Du L, Qiu L, Peng Q, Lereclus D, Zhang J, Song F et al. (2012). Identification of the promoter in the intergenic region between orf1 and cry8Ea1 controlled by sigma $\mathrm{H}$ factor. Appl Environ Microbiol 78: 4164-4168.

Flardh K, Buttner MJ. (2009). Streptomyces morphogenetics: dissecting differentiation in a filamentous bacterium. Nat Rev Microbiol 7: 36-49.

Griffin AS, West SA, Buckling A. (2004). Cooperation and competition in pathogenic bacteria. Nature 430: 1024-1027.

Guinebretière M-H, Velge P, Couvert O, Carlin F, Debuyser M-L, Nguyen-The C. (2010). Ability of Bacillus cereus group strains to cause food poisoning varies according to phylogenetic affiliation (Groups I to VII) rather than species affiliation. $J$ Clin Microbiol 48: 3388-3391.

Helgason E, Okstad OA, Caugant DA, Johansen HA, Fouet A, Mock M et al. (2000). Bacillus anthracis, Bacillus cereus, and Bacillus thuringiensis-one species on the basis of genetic evidence. Appl Environ Microbiol 66: 2627-2630.

Hernandez E, Ramisse F, Ducoureau JP, Cruel T, Cavallo JD. (1998). Bacillus thuringiensis subsp. konkukian (serotype H34) superinfection: case report and experimental evidence of pathogenicity in immunosuppressed mice. J Clin Microbiol 36: 2138-2139.

Hoffmaster AR, Ravel J, Rasko DA, Chapman GD, Chute MD, Marston CK et al. (2004). Identification of anthrax toxin genes in a Bacillus cereus associated with an illness resembling inhalation anthrax. Proc Natl Acad Sci USA 101: 8449-8454.

Kaiser D, Robinson M, Kroos L. (2010). Myxobacteria, polarity, and multicellular morphogenesis. Cold Spring Harb Perspect Biol 2: a000380.

Kho MF, Bellier A, Balasubramani V, Hu Y, Hsu W, Nielsen-LeRoux C et al. (2011). The pore-forming protein Cry5B elicits the pathogenicity of Bacillus sp. against Caenorhabditis elegans. PLoS One 6: e29122.

Kolsto AB, Lereclus D, Mock M. (2002). Genome structure and evolution of the Bacillus cereus group. Curr Top Microbiol Immunol 264: 95-108.

Lecadet MM, Blondel MO, Ribier J. (1980). Generalized transduction in Bacillus thuringiensis var. berliner 1715 using bacteriophage CP-54Ber. J Gen Microbiol 121: $203-212$.

Lereclus D, Arantes O, Chaufaux J, Lecadet M. (1989). Transformation and expression of a cloned deltaendotoxin gene in Bacillus thuringiensis. FEMS Microbiol Lett 51: 211-217.

Liu X, Wang D, Ren J, Tong C, Feng E, Wang X et al. (2013). Identification of the immunogenic spore and vegetative proteins of Bacillus anthracis vaccine strain A16R. PLoS One 8: e57959.
Lopez D, Kolter R. (2010). Extracellular signals that define distinct and coexisting cell fates in Bacillus subtilis. FEMS Microbiol Rev 34: 134-149.

Lopez D, Vlamakis H, Kolter R. (2009). Generation of multiple cell types in Bacillus subtilis. FEMS Microbiol Rev 33: 152-163.

Losick R, Desplan C. (2008). Stochasticity and cell fate. Science 320: 65-68.

Maynard Smith J, Szathmáry E. (1995). The Major Transitions in Evolution. W.H. Freeman Spektrum: Oxford, UK; New York, NY, USA.

Michod RE. (1997). Cooperation and conflict in the evolution of individuality .1. Multilevel selection of the organism. Am Nat 149: 607-645.

Michod RE. (1999). Darwinian Dynamics : Evolutionary Transitions in Fitness and Individuality. Princeton University Press: Princeton, NJ, USA; Chichester, UK.

Michod RE, Roze D. (2001). Cooperation and conflict in the evolution of multicellularity. Heredity 86: 1-7.

Moran Jr CP, Lang N, LeGrice SF, Lee G, Stephens M, Sonenshein AL et al. (1982). Nucleotide sequences that signal the initiation of transcription and translation in Bacillus subtilis. Mol Gen Genet 186: 339-346.

Muro-Pastor AM, Hess WR. (2012). Heterocyst differentiation: from single mutants to global approaches. Trends Microbiol 20: 548-557.

Ohba M, Mizuki E, Uemori A. (2009). Parasporin, a new anticancer protein group from Bacillus thuringiensis. Anticancer Res 29: 427-433.

Perchat S, Dubois T, Zouhir S, Gominet M, Poncet S, Lemy C et al. (2011). A cell-cell communication system regulates protease production during sporulation in bacteria of the Bacillus cereus group. Mol Microbiol 82: 619-633.

Priest FG, Barker M, Baillie LWJ, Holmes EC, Maiden MCJ. (2004). Population structure and evolution of the Bacillus cereus group. J Bacteriol 186: 7959-7970.

Raymond B, Bonsall MB. (2013a). Cooperation and the evolutionary ecology of bacterial virulence: the Bacillus cereus group as a novel study system. Bioessays 35: 706-716.

Raymond B, Bonsall MB. (2013b). Cooperation and the evolutionary ecology of bacterial virulence: the Bacillus cereus group as a novel study system. BioEssays 35: 706-716.

Raymond B, Davis D, Bonsall MB. (2007). Competition and reproduction in mixed infections of pathogenic and non-pathogenic Bacillus spp. J Invertebr Pathol 96: 151-155.

Raymond B, West SA, Griffin AS, Bonsall MB. (2012). The dynamics of cooperative bacterial virulence in the field. Science 337: 85-88.

Raymond B, Wyres KL, Sheppard SK, Ellis RJ, Bonsall MB. (2010a). Environmental factors determining the epidemiology and population genetic structure of the Bacillus cereus group in the field. PLoS Pathog 6: e1000905.

Raymond B, Wyres KL, Sheppard SK, Ellis RJ, Bonsall MB. (2010b). Environmental factors determining the epidemiology and population genetic structure of the Bacillus cereus group in the field. PLoS Pathog 6: e1000905.

Ross-Gillespie A, Gardner A, West SA, Griffin AS. (2007). Frequency dependence and cooperation: theory and a test with bacteria. Am Nat 170: 331-342.

Rossetti V, Schirrmeister BE, Bernasconi MV, Bagheri HC. (2010). The evolutionary path to terminal 
differentiation and division of labor in cyanobacteria. $J$ Theor Biol 262: 23-34.

Sacchi CT, Whitney AM, Mayer LW, Morey R, Steigerwalt A, Boras A et al. (2002). Sequencing of 16S rRNA gene: a rapid tool for identification of Bacillus anthracis. Emerg Infect Dis 8: 1117-1123.

Sanahuja G, Banakar R, Twyman RM, Capell T, Christou P. (2011). Bacillus thuringiensis: a century of research, development and commercial applications. Plant Biotechnol J 9: 283-300.

Sanchis V, Gohar M, Chaufaux J, Arantes O, Meier A, Agaisse $\mathrm{H}$ et al. (1999). Development and field performance of a broad-spectrum nonviable asporogenic recombinant strain of Bacillus thuringiensis with greater potency and UV resistance. Appl Environ Microbiol 65: 4032-4039.

Sandoz KM, Mitzimberg SM, Schuster M. (2007). Social cheating in Pseudomonas aeruginosa quorum sensing. Proc Natl Acad Sci USA 104: 15876-15881.

Schaeffer P, Millet J, Aubert JP. (1965). Catabolic repression of bacterial sporulation. Proc Natl Acad Sci USA 54: 704-711.

Schnepf HE, Lee S, Dojillo J, Burmeister P, Fencil K, Morera L et al. (2005). Characterization of Cry34/ Cry35 binary insecticidal proteins from diverse Bacillus thuringiensis strain collections. Appl Environ Microbiol 71: 1765-1774.

Shank EA, Kolter R. (2011). Extracellular signaling and multicellularity in Bacillus subtilis. Curr Opin Microbiol 14: 741-747.

Slamti L, Lereclus D. (2005). Specificity and polymorphism of the PlcR-PapR quorum-sensing system in the Bacillus cereus group. J Bacteriol 187: 1182-1187.

Sorokin A, Candelon B, Guilloux K, Galleron N, Wackerow-Kouzova N, Ehrlich SD et al. (2006). Multiple-locus sequence typing analysis of Bacillus cereus and Bacillus thuringiensis reveals separate clustering and a distinct population structure of psychrotrophic strains. Appl Environ Microbiol 72: 1569-1578.

Stobdan T, Kaur S, Singh A. (2004). Cloning and nucleotide sequence of a novel cry gene from Bacillus thuringiensis. Biotechnol Lett 26: 1153-1156.

Strassmann JE, Zhu Y, Queller DC. (2000). Altruism and social cheating in the social amoeba Dictyostelium discoideum. Nature 408: 965-967.

Velicer GJ, Kroos L, Lenski RE. (2000). Developmental cheating in the social bacterium Myхососcus xanthus. Nature 404: 598-601.

Vilas-Boas GT, Peruca AP, Arantes OM. (2007). Biology and taxonomy of Bacillus cereus, Bacillus anthracis, and Bacillus thuringiensis. Can J Microbiol 53: 673-687.

West SA, Griffin AS, Gardner A. (2007). Evolutionary explanations for cooperation. Curr Biol 17: R661-R672.

West SA, Griffin AS, Gardner A, Diggle SP. (2006). Social evolution theory for microorganisms. Nat Rev Microbiol 4: 597-607.

Willensdorfer M. (2009). On the evolution of differentiated multicellularity. Evolution 63: 306-323.

Yang H, Wang P, Peng Q, Rong R, Liu C, Lereclus D et al. (2012). Weak transcription of the cry1Ac gene in nonsporulating Bacillus thuringiensis cells. Appl Environ Microbiol 78: 6466-6474.

Yoshisue H, Ihara K, Nishimoto T, Sakai H, Komano T. (1995). Expression of the genes for insecticidal crystal proteins in Bacillus thuringiensis: cryIVA, not cryIVB, is transcribed by RNA polymerase containing sigma $\mathrm{H}$ and that containing sigma E. FEMS Microbiol Lett 127: $65-72$.

Zhu Y, Ji F, Shang H, Zhu Q, Wang P, Xu C et al. (2011). Gene clusters located on two large plasmids determine spore crystal association (SCA) in Bacillus thuringiensis subsp. finitimus strain YBT-020. PLoS One 6: e27164.

Supplementary Information accompanies this paper on The ISME Journal website (http://www.nature.com/ismej) 